http://jmscr.igmpublication.org/home/ ISSN (e)-2347-176x ISSN (p) 2455-0450 crossref DOI: https://dx.doi.org/10.18535/jmscr/v7i10.79

\author{
Journal Of Medical Science And Clinical Research \\ IGM Publication \\ An Official Publication of IGM Publication
}

\title{
Study of Hypofractionated Radiotherapy Regime for Locally Advanced Head and Neck Cancer
}

\author{
Authors \\ Dr Manoj Kumar Gupta1, Dr Neeti Sharma ${ }^{2}$, Dr H.S. Kumar ${ }^{3}$, Dr S.L. Jakhar \\ ${ }^{1}$ Resident Doctor, Radiotherapy Department, ATRCTRI, S.P. Medical College, Bikaner \\ ${ }^{2}$ Senior Professor and Head, Radiotherapy Department, ATRCTRI, S.P. Medical College, Bikaner \\ ${ }^{3}$ Senior Professor, Radiotherapy Department, ATRCTRI, S.P. Medical College, Bikaner \\ ${ }^{4}$ Associate Professor, Radiotherapy Department, ATRCTRI, S.P. Medical College, Bikaner
}

\begin{abstract}
The aim of this study is to evaluate the response rates and toxicity of hypofractionated radiation therapy in locally advanced head \& neck cancer patients who are not fit for concurrent chemoradiation or surgery.

Material and Methods: Thirty patients were eligible for radical treatment (metastatic excluded) were received a total dose of 50 Gray (Gy) in 16 fractions, 3.125 Gy per fraction, total treatment time 3 weeks.

Results: The overall response rates $(C R+P R)$ were $70 \%$ and Stage wise [III, IVA and IVB ] overall response were $90.9 \%, 66.66 \%$ \& $42.8 \%$. Of all primaries. The hypopharyngeal cancer patients had the worst response rates with progressive disease in 20-33\% of patients and laryngeal cancer patients having best response rates. Only one patient develop grade 4 mucositis for which he was hospitalized for conservative management. Despite the high rate of acute skin and mucosal toxicities, There were no dropouts or treatment breaks more than 7 days due to adequate nutritional and supportive management provided to the patients. The chronic grade 2 and 3 toxicities were higher for skin and mucous membrane.

Keywords: hypofractionated radiotherapy, locally advanced head and neck cancer.
\end{abstract}

\section{Introduction}

The International Agency for Research on Cancer (IARC) estimate that one-in-five men and one-insix women worldwide will develop cancer over the course of their lifetime, and that one-in-eight men and one-in-eleven women will die from their disease. A number of factors appear to be driving this increase, particularly a growing and ageing global population and an increase in exposure to cancer risk factors linked to social and economic development. Asia accounts for nearly half of the new cancer cases and more than half of cancer deaths. Estimated suggest that Asia and Africa have a higher proportion of cancer deaths $(7.3 \%$ and $57.3 \%$ respectively) compared with their incidence $\left(5.8 \%\right.$ and $48.4 \%$ respectively) ${ }^{1}$.

Head and neck cancers account for $8.04 \%$ (1.4 million) of the total cancer cases and $4.9 \%(0.47$ million) of cancer related deaths worldwide based on GLOBOCAN2018 estimates. In India, currently Head and neck cancers is the leading cause of cancer including lips and oral cavity (at top), larynx, hypopharynx, thyroid, oropharynx, salivary glands \& nasopharynx; accounting for nearly $17.74 \%$ (2.05 million) of all registered new cancer cases and related to $16.3 \%(1.27$ million) deaths in the country ${ }^{1}$. Among males, it is the leading cause of cancer mortality, accounting 
for $19.9 \%$ (0.073 million) of all male cancer deaths, while in female it accounts 5.03\%(0.0218 million) mortality. ${ }^{1}$

Nearly $60 \%$ of patients of head and neck cancer present with locally advanced but nonmetastatic disease. Locoregional failure constitutes the predominant recurrence pattern. Results of treatment of these tumors are inversely proportional to the extent of the disease ${ }^{2}$.

Squamous cell carcinoma histology in the head and neck is predominant and the primary treatment modalities for it are surgery and radiotherapy. ${ }^{3}$ Radiotherapy alone is used in nonoperable, existing co-morbidities, poor performance status, distant metastatic disease and patients who refuses surgery for the locally advanced disease. ${ }^{4,5,6}$ Radiation dose fractionation has evolved from once daily to hyperfractionation and accelerated fractionation. Nonetheless, even the most effective radiation regimens result in local controls rates of $50 \%$ to $70 \%$ and disease-free survivals of $30 \%$ to $40 \%$. So that the combined modality concurrent chemoradiotherapy is used as the standard care of treatment for locally advanced head and neck cancer. $^{7,8,9}$

The optimum treatment time for locoregional control is not clear. A reduction in the locoregional control by lengthening the treatment time has been observed and possible cause for it is radiation-induced accelerated proliferation of clonogenic tumor cells. Furthermore, reduction in the total treatment time has improved local tumor control in several clinical studies. ${ }^{10}$ A shorter treatment time may be accomplished by applying a higher dose per fraction or by increasing the daily number of radiation fractions with a minimum 6 hrs time interval between two fractions. But the short overall treatment time leads to potential increased late side effects. However, late radiation toxicity is often less relevant in patients treated in advanced stage setting due to poor overall survival.

The aim of this study is to evaluate the response rates, toxicity and survival benefits of hypofractionated radiation therapy in locally advanced head \& neck cancer patients who are not fit for concurrent chemoradiation or surgery followed by adjuvant therapy. Second aim of our study was to determine the toxicity profiles and the disease-free survival and overall survival.

\section{Materials and Method}

This was a prospective study conducted at Acharya Tulsi Regional Cancer Treatment And Research Institute, Sardar Patel Medical College and associated group of hospitals, Bikaner.

The study protocol includes 30 patients of locally advanced carcinoma of head and neck, histological proven squamous cell carcinoma, who were enrolled from June 2018 to May2019. The inclusion criteria were primary malignancies of Head and neck region i.e. starting from base of the skull to the thoracic inlet, age: $>18$ years, no prior treatment of malignancy. normal base line organ function (CBC, RFT, LFT \& others), and without Significant comorbidities.

The protocol was approved by hospital's institutional ethical committee and all patients were properly informed and consent was taken for treatment. 30 patients eligible for radical treatment (metastatic excluded), and received a total dose of 50 Gray (Gy) in 16 fractions, 3.125 Gy per fraction, total treatment time 3 weeks.

\section{BED calculation-}

$\mathrm{BED}=\mathrm{D}[1+\mathrm{d} /(\alpha / \beta)]$

Where $\mathrm{D}$ is the total dose and $\mathrm{d}$ is dose per fraction.

BED Calculation for hypofractionated arm

BED for early effects $=$

$3.125 \times 16(1+3.125 / 10)=65.625 \mathrm{~Gy}$

$\mathrm{BED}$ for late effects $=$

$3.125 \times 16(1+3.125 / 3)=102.08 \mathrm{~Gy}$

Treatment volume were included primary tumor site plus neck node region. Parallel opposed bilateral fields were planned. The dose was prescribed at midline. External beam radiotherapy was given with radiation therapy parameter on Cobalt-60 machines Theratron 780E / 780C/ 
Bhabhatron II with photon energies of $1.25 \mathrm{MeV}$. Minimum treatment distance was $>=80 \mathrm{~cm} \mathrm{SSD}$ (or SAD for iso-centric techniques).

During treatment patients were assessed for treatment response, control of symptoms and any treatment related morbidity by doing complete blood counts, biochemistry profile consisting of RFT\&LFT, chest X-ray, USG Abdomen. Nutritional status was maintained with all patients encouraged to liberal oral food intake and in the case of difficulty, the feeding tube was inserted either through the nasal route, percutaneously, or endoscopically. For patients with respiratory distress, it was sometimes elected to perform tracheostomy before starting radiation.

The primary end point of the study was the response rate (complete response (CR), partial response $(\mathrm{PR})$, and overall response rate $(\mathrm{ORR}=$ $\mathrm{CR}+\mathrm{PR})$ ) (assessment was done by WHO criteria). The secondary end points were acute (within 90 days of radiation) and late toxicity (beyond 90 days after radiation) (graded according to the RTOG/EORTC Acute and Chronic Radiation Morbidity Scoring).

Patients were evaluated 6-8 weeks after completion of treatment by the ENT surgeon and radiation oncologist. All patients underwent detailed ENT examination with a directed biopsy performed in patients with clinical suspicion of persistent primary and/or nodal disease. Wherever feasible, patients with residual disease were sent for salvage chemotherapy with combination of taxanes, platinum, and 5-fluorouracil. Salvages surgery for the removal of primary and/or nodal disease was not possible due to co-morbid conditions. After initial assessment, the patients with no evidence of residual primary and nodal disease were evaluated every 3 months till the end of the study to assess the toxicity. On subsequent follow up in 3rd, 6th month, detailed systemic examination, CBC, LFT, RFT, chest $\mathrm{x}$-ray and USG Abdomen was done to evaluate for distant metastasis and complications RT like mediastinitis, esophagitis and radiation pneumonitis.

\section{Results}

Table 1 Patient Characteristics

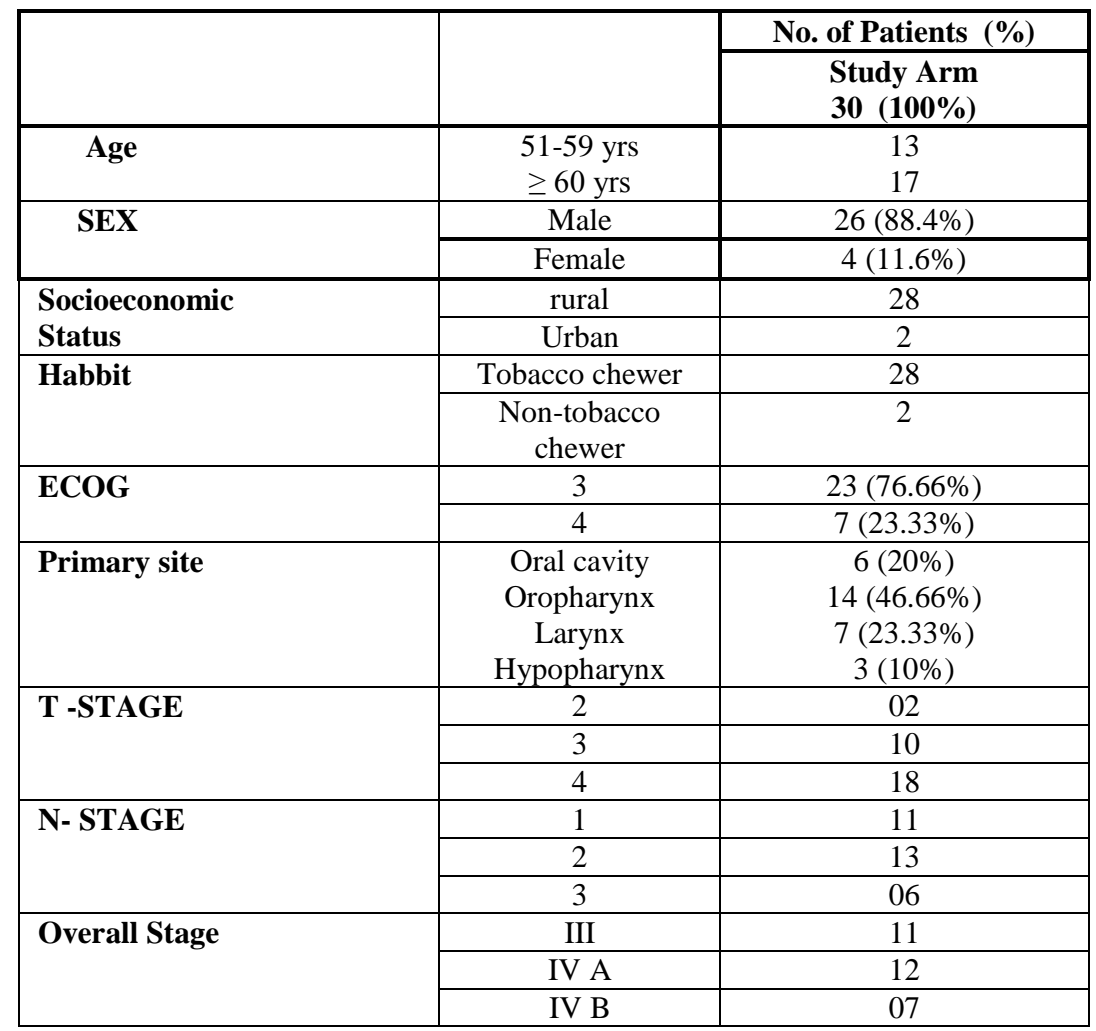


Table 2 Treatment Response

\begin{tabular}{|l|c|}
\hline & NO.OF PATIENTS (\%) \\
\cline { 2 - 2 } & STUDY ARM \\
$\mathbf{3 0}(\mathbf{1 0 0 \%})$
\end{tabular}

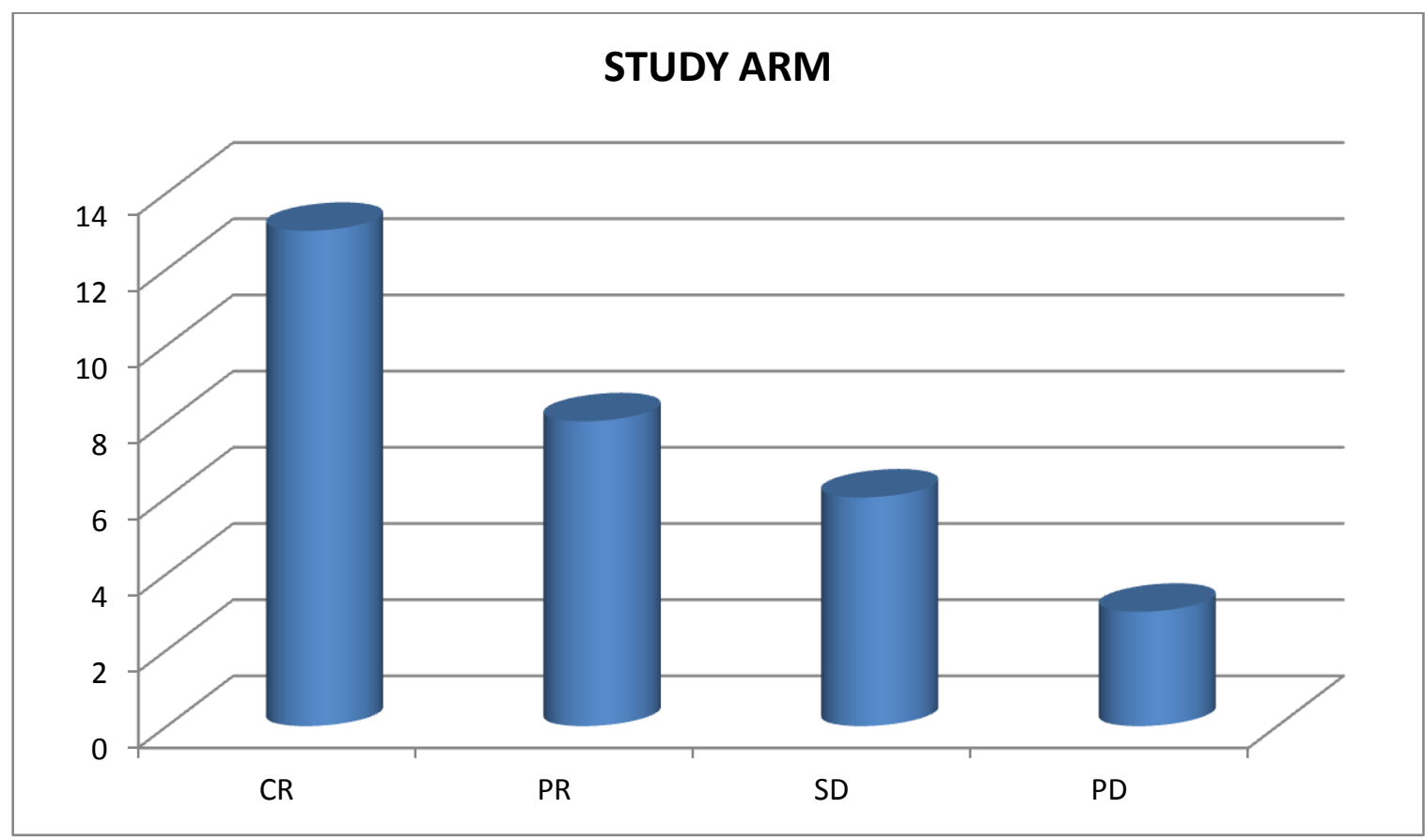

Graphical represtation of Treatment Response

Table-3 Stage Wise Response Assessment Using RECIST Criteria at the End of the Treatment

\begin{tabular}{|l|c|c|}
\hline STAGE & $\begin{array}{c}\text { RESPONSE USING WHO } \\
\text { criteria }\end{array}$ & STUDY ARM \\
\hline STAGE III & CR & $07(63.6 \%)$ \\
\cline { 2 - 3 } & PR & $03(27.2 \%)$ \\
\cline { 2 - 3 } & SD & $00(0 \%)$ \\
\cline { 2 - 3 } & PD & $01(9.1 \%)$ \\
\hline \multirow{5}{*}{ STAGE IV A } & CR & $06(50 \%)$ \\
\cline { 2 - 3 } & PR & $02(16.66 \%)$ \\
\cline { 2 - 3 } & SD & $04(33.33 \%)$ \\
\cline { 2 - 3 } & PD & $00(0 \%)$ \\
\hline & CR & $00(0 \%)$ \\
\cline { 2 - 3 } & PR & $03(42.8 \%)$ \\
\cline { 2 - 3 } & SD & $02(28.5 \%)$ \\
\cline { 2 - 3 } & PD & $02(28.5 \%)$ \\
\hline
\end{tabular}

Table-4.Toxicities

\begin{tabular}{|l|c|c|c|c|}
\hline Toxicities & $\begin{array}{c}\text { ACUTE SKIN } \\
\text { REACTION }\end{array}$ & $\begin{array}{c}\text { ACUTE } \\
\text { MUCOSITIS }\end{array}$ & $\begin{array}{c}\text { LATE SKIN } \\
\text { REACTION }\end{array}$ & $\begin{array}{c}\text { LATE MUCOUS } \\
\text { MEMBRANE REACTION }\end{array}$ \\
\hline Grade 0 & $05(16.66 \%)$ & $05(16.66 \%)$ & $08(26.66 \%)$ & $06(20 \%)$ \\
\hline Grade I & $03(10 \%)$ & $05(16.66 \%)$ & $08(26.66 \%)$ & $11(36.66 \%)$ \\
\hline Grade II & $11(36.66 \%)$ & $12(40 \%)$ & $10(33.33 \%)$ & $08(26.66 \%)$ \\
\hline Grade III & $11(36.66 \%)$ & $07(23.33 \%)$ & $04(13.33 \%)$ & $05(16.66 \%)$ \\
\hline Grade IV & $00(0.0 \%)$ & $01(03.33 \%)$ & $00(0.0 \%)$ & $00(0.0 \%)$ \\
\hline
\end{tabular}


30 patients were eligible and enrolled for this study. $88.4 \%$ \& $11.6 \%$ of patients were male and females respectively, median age 61 years (range: 50-73 years). $93.33 \%$ of individuals were tobacco chewers. Largest proportion of was oropharyngeal cancer and belongs to stage IV A, (40\%). The overall response rates $(\mathrm{CR}+\mathrm{PR})$ were $70 \%$ and Stage wise [III, IVA and IVB] overall response were $90.9 \%, 66.66 \% \& 42.8 \%$.Of all primaries, the hypopharyngeal cancer patients had the worst response rates with progressive disease in 20-33\% of patients and laryngeal cancer patients having best response rates.

Only one patient develop grade 4 mucositis for which he was hospitalized for conservative management. Despite the high rate of acute skin and mucosal toxicities, there were no dropouts or treatment breaks more than 7 days due to adequate nutritional and supportive management provided to the patients.

\section{Discussion}

A considerable number of patients with squamous cell carcinoma of the head and neck (HNSCC) are not suitable for aggressive radical treatment with surgery or chemoradiotherapy (CRT) because of a very advanced loco-regional disease, significant co-morbidities, poor performance status, distant metastatic disease, or a combination of these factors. However, this group of patients still requires some form of treatment to control their loco-regional disease and to alleviate their troublesome symptoms. ${ }^{9}$

Historically, patients with unresectable HNSCC treated by RT alone have LRC rates between 50 and $70 \%{ }^{11,12,13}$ and 5-year survival rates of 10 20\%. A large portion of these patients die of locoregional disease progression.Patients with untreated advanced stage HNSCC have a median survival of approximately 100 days.

In a curative setting, the addition of $\mathrm{CT}$ in different regimens has improved disease control and long-term survival ${ }^{14,}{ }^{16}$.RTOG $81-17$ attempted concurrent chemoradiotherapy (cisplatin based) to treat 134 patients who had unresectable carcinomas of the head and neck. ${ }^{17}$ The response and survival rates were favorable to concurrent cisplatin-based chemoradiation. Concurrent chemoradiotherapy in contrast with radiotherapy alone was suggested on the basis of other RTOG trials to treat unresectable carcinomas of the head and neck as it favors to amplify the local control for patients.

Acceleration can produce equivalent results to conventional radiation even when significant reductions in overall dose occur as demonstrated in Continuous Hyperfractionated Accelerated Radiotherapy Trial (CHART) ${ }^{18}$. 54 Gy in 36 fractions over 12 days was compared with a conventional arm of 66 Gy in 33 fractions over six-and-a-half weeks. Except for advanced laryngeal tumors there was no difference in locoregional control compared to the conventional arm. Acute morbidity was increased in CHART but the reduction in total dose and dose per fraction was associated with a reduction in later morbidities including osteochondritis, skin telangiectasia, mucosal ulceration, and laryngeal edema.

Thus Increased hyperfractionation strategy is expected to improve the probability of cancer control by delivery of a higher total dose of radiation without increasing dose for serious late normal tissue complications. Randomized trials of a 4-week hyperfractionated radiation schedule and of two other regimens of accelerated hyperfractionation were recently completed and their early results confirm to some degree the biological hypotheses on which this strategy is based. ${ }^{19}$

A small number of fractions with a larger dose per fraction is used in hypofractionated radiotherapy. The general time is normally shorter than an accelerated protocol. These regimes produce worse late effects than conventional fractionation when used in the curative setting. ${ }^{20}$ The intense responses are satisfactory if treatment volumes are kept little and tolerability can be improved by bringing treatment breaks into the protocol. 
Eight randomized trials without dose reduction and five with a total dose reduction were undertaken to perform a meta-analysis of accelerated protocols. ${ }^{21}$ The hazard ratio for death for first and second group was 0.97 (0.89 - 1.05) and 0.92 (0.86 - 0.97) respectively. The trials resulted in $2 \%$ and $1.7 \%$ respectively of the absolute survival improvement at 5 years. With that $7.3 \%$ and $2.3 \%$ improvements in locoregional control at 5 years respectively were observed too. Some accelerated form of treatment is required for the patients with inoperable conditions. Patients who do not fit to endure the burden of CRT require treatment even if it is with palliative intent to control their locoregional disease and to lessen their acute symptoms. Hypofractionated schedule is more suitable as, the treatment is completed before accelerated repopulation turns into a critical radiobiological factor. Further, the reduced number of fractions permits an increasingly effective utilization of assets, which can help avoid long waiting times for other patients and finally, it is practically required to keep the OTT as short as could be allowed for the fact that this group of patients are usually of older age and often have a poor performance status as well as significant co-morbidities. A hypofractionated schedule would be the most suitable and reasonable option from radiobiological, economic, and logistical points of view,patients having poor prognosis with a median survival of 4-8 months found fit to hypofractionated radiotherapy. ${ }^{22}$ As for these patients with poor performance status, the aim of treatment is to palliate symptoms and cause as little as possible in the ways of sideeffects.

During World War II, when RT facilities were limited the Christie Hospital in Manchester developed a 3-week schedule of RT. In one of randomized trial for locally advanced head and neck cancer the Christie hospital treated patients with hypofractionated radiation (50 - 55 Gy in 15 or 16 fractions) with concurrent single agent methotrexate (MTX) $100 \mathrm{mg} / \mathrm{m}^{2}$ given at the commencement of and after 2 weeks of a 3 -week course of treatment. Mucositis was significantly greater in the patients receiving MTX, but there was no difference in long-term toxicity. The addition of MTX increased local control from $50 \%$ to $70 \%(\mathrm{P}=0.02)$ and survival from $37 \%$ to $47 \%(\mathrm{P}=0.07)$. The significant benefit was seen among patients with oropharyngeal primaries which constituted $33 \%$ of the study population. ${ }^{23}$

To overcome accelerated repopulation of tumourclonogens to control tumour in head and neck carcinoma, it is advantageous to use shorter overall treatment time ${ }^{24}$ In SIB, the approach of using a higher dose/fraction conveniently adds the advantage of a shorter course of treatment. Overall, IMRT schedules surveyed showed a considerably shorter overall treatment time (median 39 days) than the conventional 70 Gy delivered over 7 weeks (46 days).

Various limitations affected the present investigation. It was recognized that there was selection bias in the present study due to inclusion of patients with early-stage laryngeal cancer deemed unsuitable for curative options because of high age, poor performance and/or co-morbidity. This incorporation predisposition would halfway clarify the phenomenal LRC-rates. However, most of these patients died of intercurrent disease which explains the relatively lower OS rates.

Within these confinements the present investigation gives significant information on response rates, toxicity and survival in the patients treated according to the "Christie scheme". The results referenced in this paper are helpful for clinicians to implement suitable fraction size to treat this group of patients.

Based on the encouraging results of the present examination, it is presumed that the hypofractionated scheme has radiobiological, economic and logistic advantages and can be adopted for patients with HNSCC who are unsuitable for curative treatment options as surgery or CRT. Hypofractionated schedule is considered for significant tumor regression and symptom control within a short overall treatment time (OTT) with minimal side effects. From 
radiobiological point of view, the large fraction size in a short OTT is counteracted which benefits in an increased tumor cell kill with increased potential for late side effects.

\section{References}

1. Bray F, Ferlay J, Soerjomataram I, Siegel RL, Torre LA, Jemal A. Global cancer statistics 2018: GLOBOCAN estimates of incidence and mortality worldwide for 36 cancers in 185 countries - Bray - 2018 CA: A Cancer Journal for Clinicians Wiley Online Library [Internet]. CA: A Cancer Journal for Clinicians. American Cancer Society; 2018 [cited 2019Jun28]. Available from https://onlinelibrary.wiley.com/doi/full/10. 3322/caac. 21492

2. Choudhury KB, Sharma S, Maiti S, Roy C, Mallick C. A comparison of outcomes with 'Christie Regimen' and pure accelerated radiotherapy versus conventional radiation in locally advanced squamous cell carcinoma of head and neck: A randomized controlled study. Clin Cancer Investig J [serial online] 2012 [cited 2019Jun 4]; 1:118-26. Available from: http://www.ccijonline.org/text.asp?2012/1/3/118/102878

3. Overgaard J, Hansen HS, Jørgensen K, Hjelm HM. Primary radiotherapy of larynx and pharynx carcinoma: An analysis of factors influencing local control and survival. Int $\mathbf{J}$ RadiatOncol Phys Biol 1986;12:515-21.

4. Forastiere AA, Goepfert H, Maor M, Pajak TF, Weber R, Morrison W, et al. Concurrent chemotherapy and radiotherapy for organ preservation in advanced laryngeal cancer. N Engl J Med 2003;349:2091-8.

5. Adelstein DJ, Li Y, Adams GL, Wagner $\mathrm{H}$ Jr, Kish JA, Ensley JF, et al. An intergroup phase III comparison of standard radiation therapy and two schedules of concurrent chemoradiotherapy in patients with unresectable squamous cell head and neck cancer. J ClinOncol 2003;21:92-8.

6. Bernier J. Alteration of Radiotherapy fractionation and concurrent chemotherapy; a new frontier in head and neck cancer oncology? Nat Clin Pract Oncol 20005;2:305-14.

7. Horiot JC, Bontemps $\mathrm{P}$, van den Bogaert W, Le Fur R, van den Weijngaert D, Bolla $\mathrm{M}$, et al. Accelerated fractionation (AF) compared to conventional fractionation (CF) improves loco-regional control in the radiotherapy of advanced head and neck cancers results of the EORTC 22851 randomized trial. RadiotherOncol 1997;44:111-21.

8. Overgaard J, Hansen HS, Specht L, Overgaard M, Grau C, Andersen E, et al. Five compared with six fractions per week of conventional radiotherapy of squamous cell carcinoma of head and neck DAHANCA 6 and 7 randomised controlled trial. Lancet 2003;362:933-40

9. Al-mamgani A, Tans L, Van rooij PH, Noever I, Baatenburg de jong RJ, Levendag PC. Hypofractionated radiotherapy denoted as the "Christie scheme": An effective means of palliating patients with head and neck cancers not suitable for curative treatment. Acta Oncol 2009;48:562-7.

10. Nguyen LN, Ang KK. Radiotherapy for cancer of the head and neck: Altered fractionation regimens. Lancet Oncol 2002;3:693-701.

11. Bonner JA, Giralt J, Harari PM, Ang KK, Cohen RB, Kies MS, et al. Radiotherapy plus cetuximab for squamous-cell carcinoma of the head and neck. N Engl J Med 2006;354:567-78.

12. Levendag PC, Nowak PJ, van der Sangen MJ, Jansen PP, Eijkenboom WM, Planting AS, et al. Local tumor control in radiation 
therapy of cancers in the head and neck. Am J ClinOncol 1996;19:469-77.

13. Fu KK, Pajak TF, Trotti A, Jones CU, Spencer SA, Phillips TL, et al. Radiation Therapy Oncology Group (RTOG) phase III randomized study to compare hyperfractionation and two variants of accelerated fractionation to standard fractionation radiotherapy for head and neck squamous cell carcinomasfirst report of RTOG 9003. Int J RadiatOncolBiol Phys 2000;48:7-16.

14. Laskar SG, Agarwal JP, Srinivas C, Dinsh aw KA. Radiotherapeutic management of locally advanced head and neck cancer. Expert Rev Anticancer Ther 2006; 6: 40517

15. Bonner JA, Giralt J, Harari PM, Ang KK, Cohen RB, Kies MS, et al. Radiotherapy plus cetuximab for squamous-cell carcinoma of the head and neck. N Eng J Med 2006; 354: 567-78

16. Levendag PC, Nowak PJ, van der Sangen MJ, Jansen PP, Eijkenboom WM, Planting AS, et al. Local tumor control in radiation therapy of cancers in the head and neck. Am J ClinOncol 1996; 19: 469-77

17. Kallman P, Agren A, Brahme A. Tumour and normal tissue responses to fractionated non-uniform dose delivery. Int $\mathrm{J}$ RadiatBiol 1992;62:249-62.

18. Dische S, Saunders M, Barrett A, Harvey A, Gibson D, Parmar M. A randomized multicentre trial of CHART versus conventional radiotherapy in head and neck cancer. Radiother Oncol 1997;44: 123-36.

19. Cummings BJ. Benefits of accelerated hyperfractionation for head and neck cancer. Acta Oncol 1999;38:131-6.

20. Fletcher G. Hypofractionation: lessons from complications. Radiother Oncol 1991;20:10-5.
21. Bourhis J, Le Maître A, Baujat B, Audry $\mathrm{H}$, Pignon JP. Meta-Analysis of Chemotherapy in Head, Neck Cancer Collaborative Group; Meta-Analysis of Radiotherapy in Carcinoma of Head, Neck Collaborative Group; Meta-Analysis of Chemotherapy in Nasopharynx Carcinoma Collaborative Group: A meta-analysis of updated individual patient data. Curr Opin Oncol 2007;19:188-94.

22. Kowalski L, Carvalho AL. Natural history of untreated head and neck cancer. Eur J Cancer 2000;36:1032-4.

23. Gupta NK, Pointon RC, Wilkinson PM. A randomized clinical study to constrast radiotherapy with radiotherapy and methotrexate given synchronously in head and neck cancer. ClinRadiol 1987;38:57581

24. Withers HR, Taylor JM, Maciejewski B. T he hazard of accelerated tumor clonogen repopulation during radiotherapy. Acta Oncol 1988; 27: 131-46[Taylor \& Francis Online] 